\title{
Risk of Surgical Site Infection in Patients Undergoing Total Knee Arthroplasty
}

\author{
Paulo Cezar Vidal Carneiro de Albuquerque ${ }^{1^{*}}$, Alvaro Antônio Bandeira Ferraz ${ }^{1}$, \\ Clarissa Miranda Carneiro de Albuquerque Olbertz², José Miguel Francisco da Silva Souza1, \\ Alessandro Henrique da Silva Santos ${ }^{1}$, Paula Eduarda Miranda Carneiro de Albuquerque ${ }^{3}$
}

\author{
${ }^{1}$ Federal University of Pernambuco, Recife, Brazil \\ ${ }^{2}$ Sarah Kubistckeck Hospital, Brasilia, Brazil \\ ${ }^{3}$ IMIP, Recife, Brazil \\ Email: *paulovidal_@hotmail.com
}

How to cite this paper: de Albuquerque, P.C.V.C., Ferraz, A.A.B., de Albuquerque Olbertz, C.M.C., da Silva Souza, J.M.F., da Silva Santos, A.H. and de Albuquerque, P.E.M.C. (2018) Risk of Surgical Site Infection in Patients Undergoing Total Knee Arthroplasty. Open Journal of Orthopedics, 8, 305-315.

https://doi.org/10.4236/ojo.2018.87033

Received: May 13, 2018

Accepted: July 16, 2018

Published: July 19, 2018

Copyright $\odot 2018$ by authors and Scientific Research Publishing Inc. This work is licensed under the Creative Commons Attribution International License (CC BY 4.0).

http://creativecommons.org/licenses/by/4.0/

\begin{abstract}
The aim of this study was to determine the participation of risk factors in the infection in patients who underwent total knee arthroplasty. This is a prospective longitudinal study, descriptive and analytical, that was carried out between January 2013 and December 2015. Eighty-one procedures were performed in 78 individuals, 16 males and 62 females. Three subjects underwent the procedure on both knees. The following predictive factors of infection were identified: Age, gender, comorbidities, evaluation of functional physical status using the American Society of Anesthesiologists Physical Status Classification System (ASAPS), length of hospital stay, use of prophylactic antibiotics and duration of surgery. The data was tabulated in a database using a Microsoft Excel spreadsheet. For statistical analysis, the chi-square test, chi-square test for independence and Fischer's exact test were applied, all at a significance level of 5\% (0.05). Surgical site infection was more prevalent in males (23.5\%), age over 70 years $(25.0 \%)$, for left gonarthrosis $(17.9 \%)$, in the presence of comorbidities $(17.9 \%)$, when hospital stay was 6 or more days $(27.8 \%)$, in total arthroplasties of left knee $(17,9 \%)$, in surgical times of up to 2 hours (20\%), in the use of prophylactic antibiotics (16.7\%) and in level II classification in the ASAPS (18.8\%). The independence test was not significant in any of the evaluated factors ( $p$-value greater than 0.05 ). The most prevalent bacteria identified in cases of infection was Staphylococcus aureus (33.2\%). The risk factors studied were not statistically significant.
\end{abstract}

\section{Keywords}

Knee Arthroplasty, Knee Debridement Infection, Joint Prosthetic Joint, Infection/Diagnosis, Infection Therapy 


\section{Introduction}

Healthcare-associated infection is a result of the action of microorganisms from the time of hospitalization until the time of discharge from patients of any health service. When this occurs, it increases public costs, whether through diagnoses, treatment and hospitalization, or secondary damage through labor and social leave. Surgical site infection (SSI) corresponds to infections occurring at the surgical site at incision or in manipulated tissues during the operation, and may be classified as superficial, deep and of organs and spaces [1] [2] [3] [4] [5].

The healthcare associated infection is a site specific infection criterion that occurs on or after the 3rd calendar day of admission to an important location, where day of admission is calendar day one. Site surgical infection (SSI) is defined as a microbial contamination of the surgical wound within 30 days of an operation or within 1 year after surgery, if an implant is placed in a patient [3] [5].

It is regulated in Brazil by law decree 2616/98 as a compulsory notification disease [3]. It is the second most frequent cause of infection in patients undergoing surgical treatment, with frequencies varying from $1.4 \%$ to $40 \%$, and it accounts for $17 \%$ of all infections diagnosed by health services and $11 \%$ of hospital admissions [1] [2] [3] [4] [5].

Total knee arthroplasty (TKA) is a surgical alternative for the resolution of an advanced and irreversible degenerative state of this joint. Septic case incidences vary from $0.5 \%$ to $23 \%$ [6]-[11], and mortality rates range from $0.4 \%$ to $1.2 \%$ for individuals of up to 65 years of age, and from $2 \%$ to $7 \%$ for individuals over 80 years. The most frequently found microorganisms are: Staphylococcus coagulase-negative (30\% - 43\%), Staphylococcus aureus (12\% - 23\%), Mixedflora (10\%), Streptococcus pyogenes (9\% - 10\%), Gram-Negative (3\% - 6\%) and anaerobes $(2 \%-4 \%)$. In $11 \%$ of cases, no germ is isolated [6]-[11].

The aim of this study was to determine the risk of SSI in patients submitted to TKA at the Clinic Hospital of the Federal University of Pernambuco (HC UFPE), Brazil.

\section{Material and Methods}

This study was approved by the Research Ethics Committee of Brazil's Platform under the registry of CAAE 57969416.4.0000.5208, in accordance with the guidelines of the Resolution of the National Health Council, number 466, from December 12, 2012. It is a prospective longitudinal study, that was carried out between January 2013 and December 2015, at the Clinic Hospital of the Federal University of Pernambuco (HC UFPE). The census sample was used and all the patients attended at the service during the data collection period were part of the study, which made the sample calculation dispensable since there was an interest in working with all the patients.

Study participants did not sign a consent form, as data collection was carried out in clinical follow-up charts, of patients with no alterations in medical con- 
duct, because it is a study of epidemiological data and in agreement with the ethics committee.

The study included 81 total knee arthroplasties. In total, 17 were male and 64 female. The inclusion criteria were: All arthroplasties of either gender, of all age groups, admitted for elective implant surgery. The exclusion criteria were: all surgeries previously performed in another hospital. The diagnostic criteria used for SSI was that of the National Agency of Sanitary Surveillance (ANVISA), National Healthcare Safety Network (NHSN) and American Academy of Orthopedic Surgeons Guideline (AAOS) [3] [6] [12] [13] (Figures 1-3).

Prophylaxis with Cefazoline antibiotic was routinely used during anesthetic induction. The predictive factors of infection studied were: age, gender, co morbidities, ASAPS (American Society Anesthesiologist Physical Classification), length of hospital stay, use of prophylactic antibiotics and duration of surgery [5]-[13].

\section{Data Analysis}

For data analysis, a database was elaborated on a Microsoft Excel spreadsheet, which was then exported to the SPSS software, version 18. The percent frequencies were calculated and the respective distributions constructed. For proportion comparison, the chi-square test was applied. In order to evaluate the factors that influenced patient infection, the chi-square test for independence was applied. In cases where the assumptions of the test were not satisfied, the Fisher's exact test was used. All conclusions were determined considering a significance level of $5 \%$ $(0.05)$.

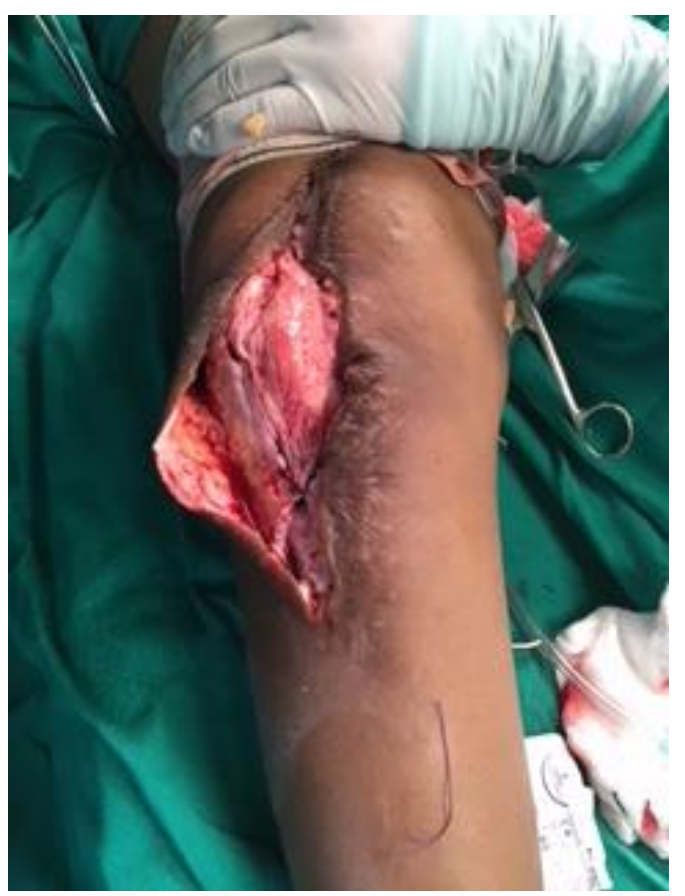

Figure 1. Knee arthroplasty infection. View of soft parts. 


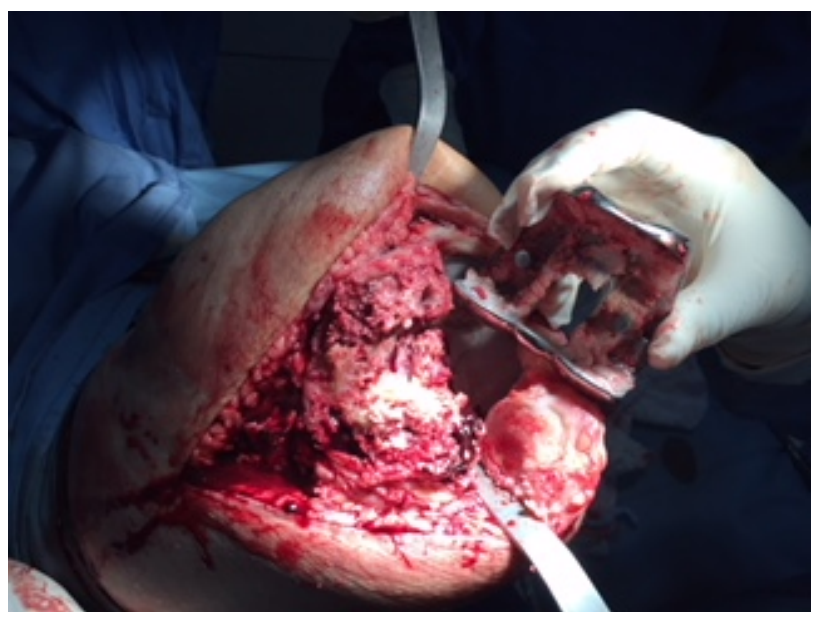

Figure 2. View of support of the femur and tibia, after removal of the prosthesis.

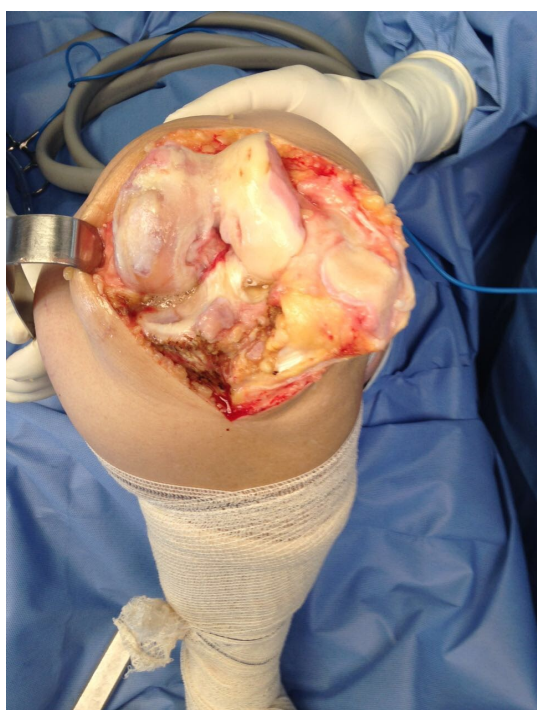

Figure 3. View of support of the femur and tibia, after removal of the prosthesis.

\section{Results}

Table 1 shows the age and sex distribution of the patients evaluated. The majority of the patients were female (79.0\%), aged 60 to 70 years (46.9\%). The proportion comparison test was significant in the two factors evaluated ( $p$-value $<0.001$ and 0.028 , respectively), indicating that these groups were relatively more frequent among the patients evaluated.

Table 2 shows the distribution of factors related to the clinical profile of patients. The majority of patients presented right gonarthrosis (52.5\%) and clinical diseases (82.7\%). Systemic arterial hypertension and Diabetes Mellitus were the most prevalent associated diseases $-59.0 \%$ and $14.0 \%$, respectively. The proportion comparison test was significant in all factors evaluated, except on the affected side ( $p$-value $=0.655)$, indicating that the number of patients with left-sided gonarthrosis is proportional to the number of patients with right-sided gonarthrosis. 
Table 1. Distribution of the personal profile of evaluated patients.

\begin{tabular}{cccc}
\hline Factor evaluated & n & $\%$ & $p_{\text {-value }}{ }^{\mathbf{1}}$ \\
\hline Gender & 17 & 21.0 & \\
Male & 64 & 79.0 & $<0.001$ \\
Female & & & \\
Age & 19 & 23.5 & \\
Less than 60 years & 38 & 46.9 & 0.028 \\
60 to 70 years & 24 & 29.6 & \\
Over 70 years &
\end{tabular}

${ }^{1} p$-valor of the chi-square test for proportion comparison.

Table 2. Distribution of the clinical profile of evaluated patients.

\begin{tabular}{cccc}
\hline Factor evaluated & n & $\%$ & p-value $^{\mathbf{1}}$ \\
\hline Principal diagnosis & 38 & 47.5 & 0.655 \\
Gonarthrosis E & 42 & 52.5 & \\
Gonarthrosis D & & & \\
Clinical diseases & 67 & 82.7 & $<0.001$ \\
Yes & 14 & 17.3 & \\
No & & & \\
Types of diseases & 59 & 59.0 & \\
SAH & 14 & 14.0 & \\
DM & 5 & 5.0 & $<0.001$ \\
Rheumatoid arthritis & 4 & 4.0 & \\
Hypothyroidism & 3 & 3.0 & \\
Arrhythmia & 2 & 2.0 & \\
Glaucoma & 2 & 2.0 & \\
SLE & 11 & 11.0 & \\
Other & & & \\
\hline
\end{tabular}

${ }^{1} p$-value of the chi-square test for proportion comparison. ${ }^{*}$ Diseases that presented unit frequency. Systemic arterial hypertension (SAH); Diabetes Mellitus (DM); Systemic Lupus Erythemasosus (SLE).

Table 3 shows the distribution of the characteristics of the surgery performed. The majority of patients remained hospitalized for 5 days or more (34.6\%), performed total right knee arthroplasty (TRKA) (52.5\%) and underwent surgery for 2 to 3 hours $(74.1 \%)$. On average, hospitalization was 7.2 days and surgery time was 2.4 hours. The proportion comparison test was significant only regarding the duration of surgery ( $p$-value $<0.001$ ), indicating that it is relatively more frequent for surgeries to last 2 to 3 hours.

Table 4 shows the distribution of factors related to infection. The majority of patients treated did not present infection (84.0\%), took a prophylactic antibiotic 
Table 3. Distribution of the characteristics of the surgery performed.

\begin{tabular}{|c|c|c|c|}
\hline Evaluated Factor & $\mathrm{n}$ & $\%$ & $p$-value ${ }^{1}$ \\
\hline \multicolumn{4}{|l|}{ Length of hospital stay } \\
\hline 4 days & 16 & 30.8 & \\
\hline 5 days & 18 & 34.6 & 0.926 \\
\hline 6 or more & 18 & 34.6 & \\
\hline Minimum-maximum & \multicolumn{2}{|c|}{$4-51$} & - \\
\hline Average \pm Standard Deviation & \multicolumn{2}{|c|}{$7.2 \pm 8.5$} & - \\
\hline \multicolumn{4}{|l|}{ Type of surgery } \\
\hline TKA_right knee & 38 & 47.5 & \multirow{2}{*}{0.655} \\
\hline TKA-left knee & 42 & 52.5 & \\
\hline \multicolumn{4}{|l|}{ Duration of surgery (in hours) } \\
\hline Up to 2 hours & 15 & 18.5 & \\
\hline Between 2 and 3 hours & 60 & 74.1 & $<0.001$ \\
\hline Above 3 hours & 6 & 7.4 & \\
\hline Minimum-maximum & \multicolumn{2}{|c|}{$1.6-3.3$} & - \\
\hline Average \pm Standard Deviation & \multicolumn{2}{|c|}{$2.4 \pm 0.4$} & - \\
\hline
\end{tabular}

${ }^{1} p$-value of the chi-square test for proportion comparison. ${ }^{\star}$ Diseases that presented unit frequency. Total Knee Arthroplasty (TKA)

Table 4. Distribution of factors related to infection and treatment of infection.

\begin{tabular}{cccc}
\hline Factor evaluated & $\mathbf{n}$ & $\%$ & $p_{\text {-value }}$ 1 $^{\mathbf{2}}$ \\
\hline Infection & 13 & 16.0 & \\
Yes & 68 & 84.0 & $<0.001$ \\
No & & & \\
PATB & 72 & 88.9 & $<0.001$ \\
Yes & 9 & 11.1 & \\
No & & & \\
ASAPS & 14 & 17.3 & $<0.001$ \\
I & 64 & 79.0 & \\
II & 3 & 3.7 & \\
III & &
\end{tabular}

${ }^{1} p$-value of the chi-square test for proportion comparison. Prophylactic antibiotic therapy (PATB). Anesthesiologist Physical Status Classification System (ASAPS: I-A normal healthy patient, II-A patient with mild systemic disease, III-A patient with severe systemic disease, IV-A patient with severe systemic disease that is a constant threat to life, V-A moribund patient who is not expected to survive without the operation, moribund patient who is not expected to survive without the operation, VI-A declared brain-dead patient whose organs are being removed for donor purposes).

(88.9\%) and were classified in group II of the ASAPS (79.0\%). The proportion-comparison test was significant in all factors evaluated $(p$-value $<0.001)$, indicating that the profile described was significantly more prevalent among the patients evaluated. 
Table 5 shows the distribution of infection according to the personal and clinical profile, as well as the treatment characteristics. A higher prevalence of infection was verified in the group of male patients (23.5\%), age over 70 years (25.0\%), left knee arthrosis (17.9\%), with comorbidities (17.9\%), length of hospital stay of 6 or more days (72.2\%), total left knee arthroplasty (17.9\%), surgical time of up to 2 hours (20\%), use of prophylactic antibiotics (16.7\%), and classification level II in the ASAPS (18.8\%). The study showed that even though a

Table 5. Distribution of infection according to personal and clinical profiles, and treatment characteristics.

\begin{tabular}{|c|c|c|c|}
\hline \multirow{2}{*}{ Evaluated factor } & \multicolumn{2}{|c|}{ Infection } & \multirow{2}{*}{$p$-value } \\
\hline & Yes & No & \\
\hline \multicolumn{4}{|l|}{ Gender } \\
\hline Male & $4(23.5 \%)$ & $13(76.5 \%)$ & \multirow{2}{*}{$0.456^{2}$} \\
\hline Female & $9(14.1 \%)$ & $55(85.9 \%)$ & \\
\hline \multicolumn{4}{|l|}{ Age } \\
\hline Less than 60 years & $1(5.3 \%)$ & $18(94.7 \%)$ & \multirow{3}{*}{$0.218^{2}$} \\
\hline 60 to 70 years & $6(15.8 \%)$ & $32(84.2 \%)$ & \\
\hline Above 70 years & $6(25.0 \%)$ & $18(75.0 \%)$ & \\
\hline \multicolumn{4}{|l|}{ Principal diagnosis } \\
\hline Left Knee Arthrosis & $7(17.9 \%)$ & $32(82.1 \%)$ & \multirow{2}{*}{$0.766^{1}$} \\
\hline Right Knee Arthrosis & $6(14.3 \%)$ & $36(85.7 \%)$ & \\
\hline \multicolumn{4}{|l|}{ Clinical diseases } \\
\hline Yes & $12(17.9 \%)$ & $55(82.1 \%)$ & \multirow{2}{*}{$0.448^{2}$} \\
\hline No & $1(7.1 \%)$ & $13(92.9 \%)$ & \\
\hline \multicolumn{4}{|l|}{ Length of hospital stay } \\
\hline 4 days & $2(12.5 \%)$ & $14(87.5 \%)$ & \multirow{3}{*}{$0.470^{2}$} \\
\hline 5 days & $2(11.1 \%)$ & $16(88.9 \%)$ & \\
\hline 6 or more & $5(27.8 \%)$ & $13(72.2 \%)$ & \\
\hline \multicolumn{4}{|l|}{ Type of surgery } \\
\hline TKA-left knee & $7(17.9 \%)$ & $32(82.1 \%)$ & \multirow{2}{*}{$0.766^{1}$} \\
\hline TKA_right knee & $6(14.3 \%)$ & $36(85.7 \%)$ & \\
\hline \multicolumn{4}{|l|}{ Time of surgery (in hours) } \\
\hline Up to 2 hours & $3(20.0 \%)$ & $12(80.0 \%)$ & \multirow{3}{*}{$0.874^{2}$} \\
\hline Between 2 and 3 hours & $9(15.0 \%)$ & $51(85.0 \%)$ & \\
\hline Above 3 hours & $1(16.7 \%)$ & $5(83.3 \%)$ & \\
\hline \multicolumn{4}{|l|}{ РATB } \\
\hline Yes & $12(16.7 \%)$ & $60(83.3 \%)$ & \multirow{2}{*}{$1.000^{2}$} \\
\hline No & $1(11.1 \%)$ & 8 (88.9\%) & \\
\hline \multicolumn{4}{|l|}{ ASAPS } \\
\hline I & $1(7.1 \%)$ & $13(92.9 \%)$ & \multirow{3}{*}{$0.673^{2}$} \\
\hline II & $12(18.8 \%)$ & $52(81.3 \%)$ & \\
\hline III & $0(0.0 \%)$ & $3(100.0 \%)$ & \\
\hline
\end{tabular}

${ }^{1} p$-value of the chi-square test for independence. ${ }^{2} p$-value of Fisher's exact test. Total knee Arthroplasty (TKN). ASAPS (American Society Anesthesiologist Functional Classification). Prophylactic antibiotic therapy (PATB). 
higher prevalence of infection was found in these groups, the independence test was not significant in any of the evaluated factors ( $p$-value greater than 0.05 ), indicating that these variables (factors) are not determinant for infection.

Table 6 shows the distribution of culture frequencies carried out on patients diagnosed with superficial and deep infections. Five cases were diagnosed as superficial infections, with a culture carried out on one patient only, without showing positivity; and eight cases were diagnosed as deep infections, with 06 cultures carried out, showing positivity in 04 patients. The Table 7 shows the distribution of frequencies of isolated bacteria in cases where the culture was positive. Staphylococcus aureus showed the highest prevalence in the cultures performed.

\section{Discussion}

In the present research study, the infection diagnosis of the surgical site obeyed the clinical, laboratory and imaging exam criteria already adopted by ANVISA, NHSN and Guiline of AAOS-and already used in other publications [1]-[6] [10] [12] [14]-[21].

Diagnose infection imaging tests were also used, including radiographs, tomography and bone scintigraphy, as well as hematological exams, including hemogram, erythrocyte sedimentation rate, c-reactive protein and bacteriological dosage-corresponding to culture positivity in 3 samples [1]-[6] [10] [14] [15].

Other factors were also used to define the cases: the presence of a communication canal with the prosthesis, two or more positive periprosthetic cultures with phenotypically identical organisms or a positive culture of a virulent organism, and the presence of pus around the prosthesis without other etiologies [1] [2] [5] [6] [10] [14] [15]-[21].

Table 6. Prevalence of positivity for the presence of bacteria.

\begin{tabular}{ccccc}
\hline Evaluated Group & $\mathbf{n}(\%)$ & Culture & $\begin{array}{c}\text { Positive in relation } \\
\text { to the group }\end{array}$ & $\begin{array}{c}\text { Positive in relation to } \\
\text { the culture }\end{array}$ \\
\hline Superficial infection & $5(38.5)$ & $1(20.0 \%)$ & $0(0.0 \%)$ & $0(0.0 \%)$ \\
Deep infection & $8(61.5)$ & $6(75.0 \%)$ & $4(50.0 \%)$ & $4(67.0 \%)$ \\
\hline
\end{tabular}

Table 7. Distribution of frequenceies of isolated bacteria in cases where the culture was positive.

\begin{tabular}{cc}
\hline Type of bacteria & $\mathbf{n}(\%)$ \\
\hline Staphylococus aureus & $2(33.2 \%)$ \\
Klebisiella pneumonie & $1(16.7 \%)$ \\
Staphylococus epidermis & $1(16.7 \%)$ \\
Staphylococus waneri & $1(16.7 \%)$ \\
Salmonella ssp & $1(16.7 \%)$ \\
\hline
\end{tabular}


The highest prevalence of infection found was in males and in the left knee. These findings were not found in other studies. However, older patients are more prone to this complication [2] [5] [8] [9] [11]-[16] [18] [19] [20] [21].

Diabetes mellitus, with index obesity greater than 40 , presented 3.3 and 3.1 times greater chance of TKA infection [6]-[20]. In our results, we were not able to repeat the obesity index because this condition is not available. Diabetes Mellitus was also the second most frequent condition, however, it was not a statistically significant factor for increased risk characterization.

The use of antimicrobial prophylaxis, care in preparing the patient's skin before the operation and use of laminar flow in surgical theaters, have reduced intra operative contamination rates [1] [2] [6] [10]-[21]. In this study, skin decontamination and the use of prophylactic antibiotics were carried out before anesthetic induction.

According to Pinto et al the infection rate in total primary knee arthroplasty may reach $6 \%$ and in revision surgeries $14 \%$ [6]. In this study, the infection rate of $16.4 \%$ in the presented casuistry was much higher than that found in other clinical studies. It was important to evaluate whether the categorical variables studied are responsible for accentuating and maintaining the risk of infection in our environment. The elevated infection rates found in this study differed from several other previous studies [7] [8] [9] [10] [12]-[21].

Malinzak et al., reported an infection rate of $0.51 \%$ in 8494 hip and knee arthroplasties [20]. Among the reported risk factors were: precocious age, Diabetes Mellitus, smoking, malnutrition, poor anticoagulation control, cancer and patients with a body mass index above 40 . These groups presented a three-fold higher risk for SSI [7]-[14] [18] [19] [20] [21].

Prolonged surgical time may increase the period of contamination and cellular and local damage, through bleeding, cauterization and suturing of the wound or a combination of these [2] [15]-[21]. The study also found a higher frequency of infection in patients with associated diseases, hospital stay of more than 6 days, surgical time of between 2 and 3 hours, use of prophylactic antibiotics and in patients classified as type II in the classification of ASAPS. However, in this study the results were not significant for hospital stays higher or lower than 5 days, and the other variables studied (duration of surgery, associated comorbidities, use of prophylactic antibiotic, ASAPS classification, side of operated knee, age and length of hospital stay) were also not significant and determinant factors for increased prevalence of infection, contrasting with other studies [2]-[12] [14]-[21].

Late infections had frequent agent specimens such as Cultibacterium, Coagulase-negative Staphylococcus and Staphylococcus aureus. These were associated with comorbidities [10] [11] [12] [13] [14]. In the present study, positive culture of these bacteria was not associated to comorbidities [7]-[21].

A small proportion of individuals diagnosed with arthroplasty infection maintained the result of negative culture and required empirical therapy, corroborating with other authors [10] [11] [12] [13] [14]. In this study the negative 
bacteriological culture rate was 5\%. Biofilm increases bacterial resistance to the microbial agent and makes difficult an exclusively antimicrobial treatment. This was also observed in patients of this present study and in these cases it is necessary to remove the implants [7]-[14].

These results showed that the risk factors studied, associated with the infections of patients submitted to TKA in this environment, do not justify high frequencies of SSI. This suggests a need for carrying out new studies aimed at other variables, including those related to environmental control, such as sterilization and handling of surgical instrumentation materials, asepsis condition and other human resources involved.

\section{Conclusion}

It is possible to conclude from this study that the risk factors considered were not statistically significant for the occurrence of surgical site infection, in patients submitted to total knee arthroplasty at the Clinic Hospital of the Federal University of Pernambuco.

\section{References}

[1] Padoveze, M.C. and Branco, C.C.M. (2014) Infections Related to Health Care: Cahllenges to Public Health in Brazil. Revista de Saúde Pública, 48, 995-1001. https://doi.org/10.1590/S0034-8910.2014048004825

[2] Ercole, F.F., Franco, L.M.C.F., Macieira, T.G.R., Wenceslau, L.C.C., Resende, H.I.N. and Chianca, T.C.M. (2011) Risk of Surgical Site Infection in Patients Undergoing Orthopedic Surgery. Revista Latino-Americana de Enfermagem, 19, 1362-1368.

[3] ASA Physical Status Classification Guideline December 30, 2017. http://www.cpsbc.ca/files/pdf/NHMSFAP-ASA-Physical-Status-Classification.pdf

[4] Ministério da Saúde. Agência Nacional de Vigilância Sanitária (BR). Surgical site. Criteria for Infections Related Health Care. Ministério da Saúde, Brasilia (FD), 2009. 19 p. http://www.anvisa.gov.br

[5] Amaradeep, G., Prakah, S.S.S. and Manjappa, C.N. (2017) Surgical Site Infections in Orthopedic Implant Surgery and Its Risk Factors: A Prospective Study in Teaching Hospital. International Journal of Orthopaedics Sciences, 3, 169-172.

[6] (2001) National Nosocomial Infections Surveillance System (NNIS) System Report. Data Summary from January 1992-June 2001. American Journal of Infection Control, 29, 404.

[7] Pinto, C.Z.S., Alpendre, F.T., Stier, C.J.N., Maziero, E.C.S., Alencar, P.G.A. and Cruz, E.D.A. (2015) Characterization of Hip and Knee Arthroplasties and Factors Associated with Infection. Revista Brasileira de Ortopedia, 50, 694-699.

[8] Oppermann, C.M., Caregnato, R.C.A. and Azambuja, M.S. (2017) Risks of Infection in Heath Services outside the Hospital Context: Integrative Review. Vol. 7, No. 3.

[9] Junior, L.H.C., Temponi, E.F. and Badet, R. (2013) Infection in Total Knee Arthroplasty: Diagnosis and Treatment. Revista Brasileira de Ortopedia, 48, 389-396. https://doi.org/10.1016/j.rbo.2013.01.003

[10] Lima, A.L.M. and Oliveira, R.D. (2010) Update on Joint Prosthesis Infections. Revista Brasileira de Ortopedia, 45, 520-3.

http://producao.usp.br/handle/BDPI/10291 
[11] Pradella, J.G.D.P., Bovo, M., Salles, M.J.C., Klautauc, G.B., Camargo, O.A.P. and Curye, R.P.L. (2013) Primary Arthroplasty of Infected Knee: Risk Factor for Failure in Surgical Therapy. Revista Brasileira de Ortopedia, 48, 432-437. https://doi.org/10.1016/j.rbo.2012.10.007

[12] Ferreira, M.C., Oliveira, J.C.P., Zidan, F.F., Franciozi, C.E.S., Luzo, M.V.M. and Abdalla, J.R. (2017) Total Knee and Hip Arthroplasty; the Worrying Reality of Care in the Brazilian Unified Health System. Revista Brasileira de Ortopedia.

[13] Osmon, D.R., Berbari, E.F. and Berendt, A.R. (2013) Executive Summary: Diagnosis and Management of Prosthetic Joint Infection: Clinical Practice Guidelines by the Infectious Diseases Society of America. Clinical Infectious Diseases, 56, 1. https://doi.org/10.1093/cid/cis966

[14] Souza, J.M.S., Ferreira, R.S., Lima, A.J.P., Filho, A.C.P.S. and Albuquerque, P.C.V.C. (2016) Clinical Demographic Study of Total Knee Arthroplasty in University Hospital. Acta Ortopédica Brasileira, 24, 300-303. https://doi.org/10.1590/1413-785220162406159988

[15] Workgroup Convened by the Musculosskeletal Infection Society (2011) New Definition for Periprosthetic Joint Infection. The Journal of Arthroplasty, 26, 1136. https://doi.org/10.1016/j.arth.2011.09.026

[16] Mortazavi, S.M., Schwartsenberg, J., Austin, M.S., Purtil, J.J. and Parvizi, J. (2010) Revision Total Knee Arthrosplasty Infection; Incidence and Predictors. Clinical Orthopaedics and Related Research, 468, 2052-2059. https://doi.org/10.1007/s11999-010-1308-6

[17] Naranje, S., Lendway, L., Mehle, S.B.S. and Gioe, T.J. (2015) Does Operative Time Affect Infection Rates in Primary Total Knee Arthroplasty. Clinical Orthopaedics and Related Research, 473, 64-69. https://doi.org/10.1007/s11999-014-3628-4

[18] Jamsen, E., Huhtala, H., Puolakka, T. and Moilanen, T. (2009) Risk Factors for Infection after Knee Arthroplasty. A Register-Based Analysis of 43,149 Cases. The Journal of Bone and Joint Surgery, 91, 38-47. https://doi.org/10.2106/JBJS.G.01686

[19] Babkin, Y., Raveh, D., Lifschitz, M., Itzchaki, M., Wiener-Well, Y., Kopuit, P., Jerassy, Z. and Yinnon, A.M. (2007) Incidence and Risk Factors for Surgical Infection after Total Knee Replacement. Scandinavian Journal of Infectious Diseases, 39, 890-895. https://doi.org/10.1080/00365540701387056

[20] Kilgus, D.J., Howe, D.J. and Strang, A. (2002) Results of Periprosthetic Hip and Knee Infections Caused by Resistant Bacteria. Clinical Orthopaedics and Related Research, 404, 116-124. https://doi.org/10.1097/00003086-200211000-00021

[21] Malinzak, R.A., Ritter, M.A., Berend, M.E., Meding, J.B., Olberding, E.M. and Davis, K.E. (2009) Morbidly Obese, Diabetic, Younger, an Unilateral Joint Arthroplasty Patients Have Elevated Total Joint Arthrosplasty Infection Rates. The Journal of Arthroplasty, 24, 84-88. https://doi.org/10.1016/j.arth.2009.05.016 